\title{
Autonomous Driving Strategies at Intersections: Scenarios, State-of-the-Art, and Future Outlooks
}

\author{
Lianzhen Wei, Zirui Li, Jianwei Gong, Cheng Gong, Jiachen Li
}

\begin{abstract}
Due to the complex and dynamic character of intersection scenarios, the autonomous driving strategy at intersections has been a difficult problem and a hot point in the research of intelligent transportation systems in recent years. This paper gives a brief summary of state-of-the-art autonomous driving strategies at intersections. Firstly, we enumerate and analyze common types of intersection scenarios, corresponding simulation platforms, as well as related datasets. Secondly, by reviewing previous studies, we have summarized characteristics of existing autonomous driving strategies and classified them into several categories. Finally, we point out problems of the existing autonomous driving strategies and put forward several valuable research outlooks.
\end{abstract}

\section{INTRODUCTION}

As a typical traffic scenario, the intersection plays an important role but also brings serious problems, which have become the focus of many researchers. According to the U.S. National Highway Traffic Safety Administration's fatality analysis report, more than one-fourth of fatal crashes in the U.S. occur at or are related to the intersection, and about $50 \%$ of these occur at uncontrolled intersections [1]. In addition to frequent safety-related accidents, intersection congestion often occurs, which brings huge economic loss and environmental pollution around the world.

A great many efforts have been done in different aspects to tackle the above problems. According to the strategy implemented, solutions can be roughly classified into two categories: (a) research about the traffic structure; (b) research about the autonomous vehicle [2]. The first kind of research focuses on the topological characteristic and the signal control method at intersections. Adopting different intersection topologies in different situations is proved to be an effective measure to dredge traffic flow and ensure interaction safety [3]. Also, some researchers focus on optimizing the control of traffic lights to improve the efficiency of the intersection. Lian et al. have proposed two adaptive signal control algorithms which are effective for easing traffic congestion and achieve adaptive signal control objectives using real-time traffic information [4]. Soheil et al. have proposed an adaptive traffic signal controller based on reinforcement learning, which can

*This research was supported by the National Natural Science Foundation of China under grant number U19A2083.

Lianzhen Wei, Zirui Li, Jianwei Gong, Cheng Gong are from the School of Mechanical Engineering, Beijing Institute of Technology, Beijing, China. (E-mails: 3120200396@bit.edu.cn; 3120195255@bit.edu.cn; gongjianwei @ bit.edu.cn; chenggong@bit.edu.cn). Jiachen Li is with University of California, Berkeley, USA (E-mail: jiachen_li@berkeley.edu).

Zirui Li is also from the Department of Transport and Planning, Faculty of Civil Engineering and Geosciences, Delft University of Technology, Stevinweg 1, 2628 CN Delft, The Netherlands.

(Corresponding author: Jianwei Gong and Zirui Li) receive non-preset high-dimensional sensory information and perform self-learning to minimize the delay at intersections [5]. Although these signal control strategies can partially improve the traffic flow if all approaches to the intersection are not equally congested, they cannot eliminate the stop delay of vehicles at intersections regardless of the traffic volume [6]. With the development of intelligent network communication as well as autonomous driving algorithm, autonomous driving technology has become an important way to solve the above problems. Some researchers focus on the autonomous driving strategy at intersections to improve traffic efficiency and reduce pollution to a greater extent.

With the accumulation of research on intersection driving strategies, it is time to classify and organize them. Throughout the past, we can see several overviews about intersections or about driving strategies, but the two are not well integrated. For instance, overviews on the intersection monitoring or the scheduling are presented in [7-9]. However, these studies rarely analyze and solve intersection problems from the perspective of autonomous driving vehicles. There are also some reviews on the decision-making for autonomous driving vehicles in $[2,10]$. However, these studies only focus on autonomous driving vehicles and have not made an analysis for the specific scenario of intersections. Different from the above reviews, we narrow the scope of our research and focus on the autonomous driving strategy at intersections. In addition to summarizing autonomous driving strategies of the intersection scenario, this paper also contains the following features: 1) The categories of existing intersection scenarios are divided, and the characteristics of intersection scenarios are introduced. 2) The simulation platforms and datasets related to the intersection have been summarized. 3) The inadequacy of the existing autonomous driving strategies at intersections is analyzed, and future outlooks are forecasted.

The rest of this paper is organized as follows. Section II presents a detailed introduction of the outline, including different types and characteristics of intersections, simulation platforms, as well as related datasets. Section III summarizes state-of-the-art autonomous driving strategies at intersections into several categories and analyzes each type of strategy. Section IV points out the inadequacy of existing autonomous driving strategies and puts forward future outlooks. Finally, we make a general conclusion about the full text in section $\mathrm{V}$.

\section{THE OUTLINE OF INTERSECTIONS}

In order to show a comprehensive and detailed description of intersection scenarios, we have summarized an outline in this section. It includes the common types and characteristics of intersections, corresponding simulation platforms, as well as related open source datasets. 


\section{A. Types and Characteristics of Intersections}

Autonomous driving at intersections is difficult mainly due to complex traffic conditions. Firstly, there are different types of participants, such as vehicles, motorcycles, bicycles, and pedestrians. Their movements are sometimes irregular and unpredictable. Secondly, The intersection structure itself

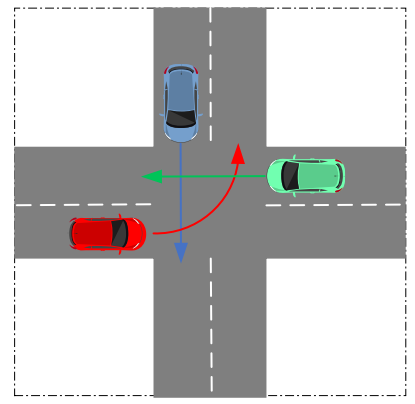

(a) Crossroad

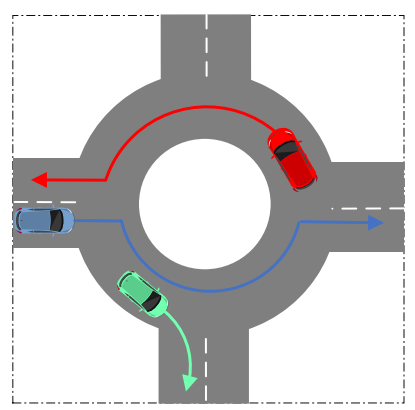

(e) Roundabout

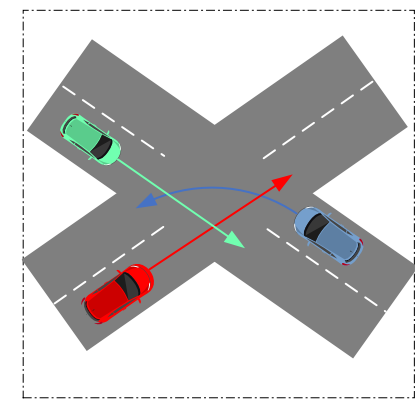

(b) X-intersection

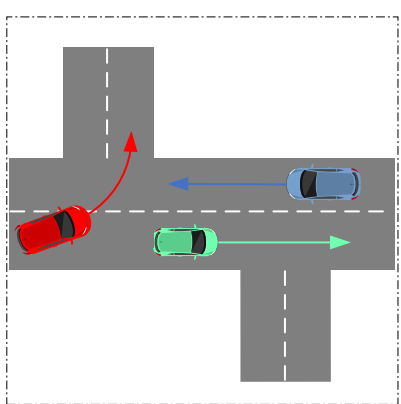

(f) Misaligned intersection

is very complicated in terms of topology. Here, we can classify intersections at grade into several main categories based on their topology: crossroad, X-intersection, Y-intersection, T-intersection, roundabout, misaligned intersection, ramp merge, and deformed intersection. These intersections are illustrated in Figure 1.

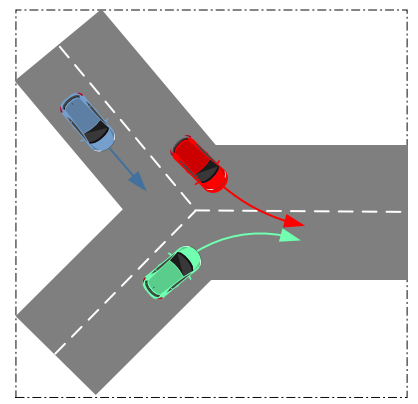

(c) Y-intersection

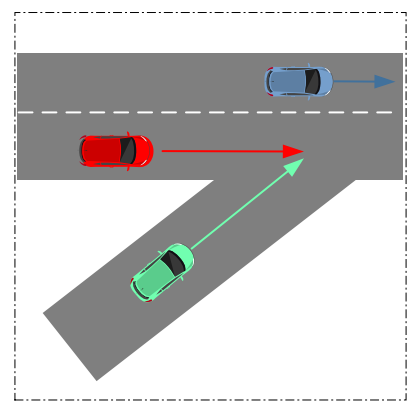

(g) Ramp merge

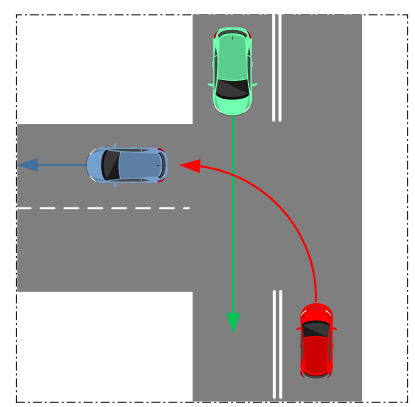

(d) T-intersection

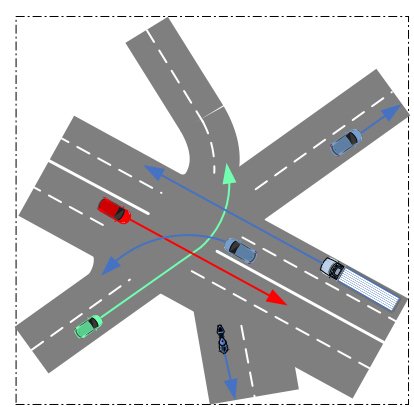

(h) Deformed intersection

Figure 1. Different types of intersections

The main difference between intersections and other road structures is that there are more conflict points at intersections [11]. So many conflict points lead to a greater probability of collisions between vehicles. In particular, the left turn of vehicles is more likely to collide than the straight or the right turn [12]. To improve the safety and efficiency of vehicles passing through the intersection, many researchers have made contributions. Among them, there are many simulations that can be applied to intersection algorithm testing, valuable datasets, as well as a variety of driving strategies. From the perspective of driving strategy, the current research pays more attention to the autonomous driving algorithm of the isolated intersection, such as $[13,14]$. In fact, vehicles in the road network will pass through multiple intersections in turn, and vehicles at multiple intersections are relevant in the long term. Considering that the optimization of a single intersection will finitely promote the overall traffic flow, Some scholars have focused on the global collaborative optimization of multiple intersections such as $[15,16]$.

\section{B. Simulation Platforms}

To shorten the time cost, reduce the economic cost and ensure safety, the training and testing of autonomous driving strategy usually start from simulation platforms. Here, we have summarized the commonly used simulation platforms for autonomous driving strategies at intersections in Table I. Some of them are commercial, while the others are open source and can be used for free.
Different simulation platforms have different focuses and we divide them into three sub-categories. Well, the barriers between simulation platforms are gradually disappearing. To make the simulation results closer to the real effect, co-simulation is becoming more and more common. Also, the combination of real datasets and real maps in the simulation platform is underway.

\section{1) Special Scene Simulation Platforms}

Some simulation platforms like FLUIDS focus on the simulation of the special scene including intersections. These simulation platforms have a small scope of application, but have strong pertinence and are very suitable for intersection research.

\section{2) Traffic Flow Simulation Platforms}

Some simulation platforms like PTV Vissim and SUMO focus on the traffic flow modeling of intelligent transportation systems. These platforms can be combined with real maps to provide more complete traffic road information for traffic flow control.

\section{3) Autonomous Driving Vehicle Simulation Platforms}

Some simulation platforms like CARLA, PreScan, and Carsim focus on the simulation of autonomous driving vehicles. They provide predetermined maps, vehicle models, as well as sensor models. These simulation platforms usually have rendering engines like Unreal Engine or Unity to simulate the external environment more realistically. 
TABLE I. CORRESPONDING SIMULATION PlatFormS

\begin{tabular}{|c|c|c|c|c|c|}
\hline Simulator & Provider & Supported Systems & Type & $U R L$ & Reference \\
\hline FLUIDS & Berkeley AUTOLAB & Linux & Open Source & https://urban-driving-simulator.readthedocs.io/ & [17] \\
\hline PTV Vissim & PTV Group & Windows, Linux & Commercial & https://www.ptvgroup.com/en/solutions/products/ptv-vissim/ & [18] \\
\hline SUMO & Eclipse Foundation & Windows, Linux, macOS & Open Source & https://www.eclipse.org/sumo/ & [19] \\
\hline PreScan & TASS International & Windows & Commercial & https://tass.plm.automation.siemens.com/prescan/ & [20] \\
\hline CARLA & $\mathrm{CVC}$ & Windows, Linux & Open Source & https://carla.org/ & [21] \\
\hline Carsim & Mechanical Simulation & Windows, Linux & Commercial & https://www.carsim.com/products/carsim/ & [22] \\
\hline SUMMIT & AdaCompNUS & Linux & Open Source & https://adacompnus.github.io/summit-docs/ & [23] \\
\hline LGSVL & LG Electronics & Windows, Linux & Open Source & https://www.svlsimulator.com/ & [24] \\
\hline Apollo & Baidu & Windows, Linux & Open Source & https://apollo.auto/gamesim.html & [25] \\
\hline AirSim & Microsoft & Windows, Linux, macOS & Open Source & https://microsoft.github.io/AirSim/ & [26] \\
\hline VTD & Hexagon & Linux & Commercial & https://vires.mscsoftware.com/ & [27] \\
\hline 51Sim-One & 51WORLD & Windows, Linux & Commercial & https://www.51aes.com/values/simulation & [28] \\
\hline ASM Traffic & dSPACE & Windows & Commercial & $\begin{array}{c}\text { https://www.dspace.com/en/pub/home/medien/product_info } \\
\text { /prodinf_asm_traffic.cfm }\end{array}$ & [29] \\
\hline DYNA4 & VECTOR & Windows & Commercial & $\begin{array}{l}\text { https://www.vector.com/int/en/products/products-a-z/ } \\
\text { software/dyna4/ }\end{array}$ & [30] \\
\hline $\begin{array}{l}\text { SCANeR } \\
\text { Studio }\end{array}$ & AVSimulation & Windwos & Commercial & https://www.avsimulation.com/scaner-studio/ & [31] \\
\hline PanoSim & PanoSim Technologies & Windwos & Commercial & http://www.panosim.com/ & [32] \\
\hline MATLAB & MathWorks & Windwos & Commercial & https://www.mathworks.com/products/matlab.html & [33] \\
\hline
\end{tabular}

\section{Related Datasets}

Datasets can be used for driving strategy training or verification, which is of great significance for the development of intelligent transportation systems and the progress of autonomous driving technologies. Over the past decade, a number of datasets for autonomous driving have been made public by numerous institutions around the world.

According to the purpose of datasets, they can be divided into object recognition, object tracking, road/lane detection, semantic segmentation, behavior analysis, end-to-end learning, etc. Junyao Guo et al. have sorted out existing autonomous driving datasets and classified them according to autonomous driving tasks [34]. They have summarized a lot of datasets, but these datasets are not specifically for intersection scenarios. Here, we have combined several reviews of datasets and newly released datasets to specifically list some of those that are highly related to intersections. To facilitate the acquisition of datasets, we have given their links. In addition, we also shared a dataset website which is maintained by GRAVITI: https://gas.graviti.cn/open-datasets.

Among them, some datasets are specifically for scenarios of intersections like ACFR. There are also some datasets related to traffic sign recognition like Tsinghua-Tencent $100 \mathrm{~K}$. These datasets are helpful for training autonomous vehicles to recognize traffic signs at intersections. In addition, some newly released datasets can support the completion of rich training or testing tasks, such as object recognition and tracking, semantic understanding, lane/road detection, pedestrian prediction, etc. These datasets are also of great significance in intersection research.

TABLE II. RELATED OPEN SOURCE DATASETS

\begin{tabular}{|c|c|c|c|c|}
\hline Name & Provider & Data Contents & URL & Reference \\
\hline ACFR & $\begin{array}{c}\text { The University of } \\
\text { Sydney }\end{array}$ & Vehicle trajectories at five roundabouts & $\begin{array}{c}\text { http://its.acfr.usyd.edu.au/datasets/ } \\
\text { five-roundabouts-dataset/ }\end{array}$ \\
\hline MTID & BEUMER Group & Intersection scenario data & https://vap.aau.dk/dataset/ \\
\hline $\begin{array}{c}\text { Tsinghua-Tencent } \\
\text { 100K }\end{array}$ & Tsinghua; Tencent & Traffic sign recognition & https:///cg.cs.tsinghua.edu.cn/traffic-sign/ \\
\hline MTSD & Mapillary & Traffic sign recognition & [37] \\
\hline $\begin{array}{c}\text { ApolloScape } \\
\text { Mapillary Vistas } \\
\text { Dataset }\end{array}$ & Mapillary & $\begin{array}{c}\text { Trajectory prediction; 3D lidar object detection and } \\
\text { tracking; Stereo estimation; Lanemark segmentation; } \\
\text { Online self-localization; 3D car instance understanding }\end{array}$ & http://apolloscape.auto/ \\
\hline [38] & Visual road-scene understanding & http://eval-vistas.mapillary.com/ \\
\hline
\end{tabular}




\begin{tabular}{|c|c|c|c|c|}
\hline KITTI & KIT; TTIC & $\begin{array}{c}\text { Odometry; Object detection; Tracking benchmarks; } \\
\text { Road benchmark }\end{array}$ & http://www.cvlibs.net/datasets/kitti/ & [41] \\
\hline Cityscapes & $\begin{array}{c}\text { Daimler AG R\&D; } \\
\text { Max Planck Institute; } \\
\text { TU Darmstadt }\end{array}$ & Semantic understanding of urban street scenes & https://www.cityscapes-dataset.com/ & [42] \\
\hline JAAD & Yorku EECS & Object detection and tracking; Behavior analysis & http://data.nvision2.eecs.yorku.ca/JAAD_dataset/ & [43] \\
\hline BDD100K & $\begin{array}{l}\text { The University of } \\
\text { Berkeley }\end{array}$ & $\begin{array}{l}\text { Object detection and tracking; Semantic segmentation; } \\
\text { Lane/road detection }\end{array}$ & https://bdd-data.berkeley.edu/ & [44] \\
\hline H3D & $\begin{array}{l}\text { Honda Research } \\
\text { Institute }\end{array}$ & Object detection and tracking & http://usa.honda-ri.com/H3D & [45] \\
\hline nuScenes & Motional & $\begin{array}{l}\text { Object detection and tracking; Semantic segmentation; } \\
\text { Object prediction }\end{array}$ & https://www.nuscenes.org/ & [46] \\
\hline
\end{tabular}

\section{REVIEW OF DRIVING STRATEGIES AT INTERSECTIONS}

In order to improve traffic efficiency and reduce the pollution caused by vehicle delay to a greater extent, numerous researchers focus on the autonomous driving strategy at intersections. In this chapter, we make a concise summary of state-of-the-art autonomous driving strategies at intersections. Firstly, the autonomous driving strategy is divided into the cooperative driving strategy and the individual driving strategy. The cooperative driving strategy can be divided into the centralized driving strategy and the distributed driving strategy while the individual driving strategy can be divided into the classical driving strategy and the learning-based driving strategy. Each strategy includes many specific methods, as shown in Figure 2.

\section{A. The Cooperative Driving Strategy}

The core idea of cooperative driving is to construct and execute a global optimal sequence for vehicles [2]. According to the architecture of computing and control, we divide the cooperative driving strategy into two types: the centralized driving strategy and the distributed driving strategy.

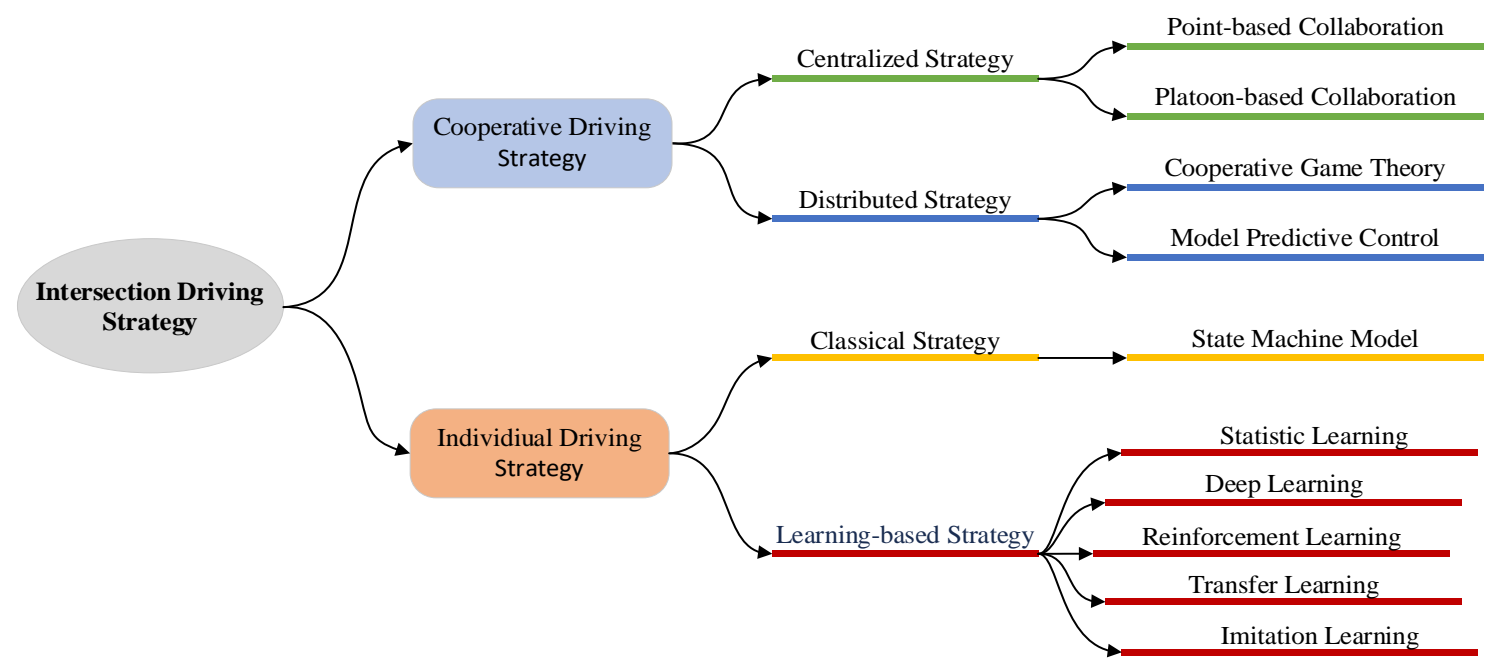

Figure 2. State-of-the-art autonomous driving strategies at intersections

\section{1) The Centralized Driving Strategy}

The centralized driving strategy mainly relies on vehicle-to-infrastructure (V2I) technology to achieve two-way communication between vehicles and road infrastructures. Obviously, the centralized driving strategy requires the intersection coordination unit (ICU) to schedule vehicles in a certain range around the intersection [6]. In the process of interaction, vehicles are supposed to send their information, such as location, speed, driving intention, etc. to the ICU. Then, the ICU carries out the centralized control through a specific scheduling algorithm and sends the high-level control information to vehicles. Vehicles can receive the high-level command and carry out the low-level control individually. The existing centralized cooperative driving strategy can also be divided into two the point-based collaboration method and the platoon-based collaboration method. The former treats each vehicle as a specific point for priority division, and the latter first groups the vehicles, and then coordinates the passing order of different groups.

The point-based collaboration means that the ICU assigns different priorities to individual vehicles near the intersection in some way. The vehicle with higher priority passes first, and the vehicle with lower priority passes later. If the time difference for two vehicles reaching the conflict point were less than the threshold, the longitudinal speeds of the two vehicles would be adjusted to ensure safety. There are many ways to divide vehicle priority like the rule-based method, the search-based method, the optimization-based method, and so on. In [14], a new method based on heuristic rules and Monte Carlo tree search is proposed, which can obtain a suboptimal vehicle sequence in a short time. In [47], a priority-based traffic architecture is proposed. The ICU gives priority to all intersection participants based on specific rules. In [48], the multi-vehicle traffic problem is modeled as a multi-objective 
optimization problem. The objective function includes delay time, fuel consumption, comfort, and other indicators. The constraint condition is usually the allowable speed range of vehicles and the time difference reaching the conflict point. The acceleration or deceleration of the vehicle can be used as the control quantity. In [49], two optimal allocation methods of vehicle traffic sequence are studied, which are dynamic programming and Petri Nets model.

The platoon-based collaboration method groups vehicles into several platoons before they arrive in the vicinity of the intersection. vehicles pass through the intersection safely and efficiently according to the set flow queue mode. In [50], vehicles arriving at the intersection are divided into discrete groups, and the continuous traffic flow problem is simplified to a multi-vehicle cooperation problem with limited vehicles. In [51], a cooperative framework is proposed. The scheduling algorithm frequently plans strategies according to the real traffic situation to guide vehicle rearrangement. In [52], a hierarchical centralized control strategy is proposed. Firstly, vehicles are listed as the standard traffic flow queue before they arrive at the intersection. Secondly, the ICU uses the optimal control method to deal with the traffic queue. It is worth mentioning that the optimal control operation can be carried out offline in advance, while the online matching is carried out according to the actual situation. In [53], a platoon-based cooperative strategy for mixed autonomous and human-driven vehicles at intersections is proposed. It assumes that an autonomous vehicle is leading, and other hybrid vehicles follow behind. This orderly arrangement of traffic can reduce energy consumption. In summary, the centralized driving strategy can achieve global optimization [14]. However, the amount of calculation will increase significantly with the increase of vehicle numbers, which brings a huge challenge to solution calculation.

\section{2) The Distributed Driving Strategy}

The distributed driving strategy uses vehicle-to-vehicle (V2V) technology to communicate with each other between vehicles to avoid collisions. Compared with the centralized driving strategy, it has the following advantages. 1) No ICU is required, so the economic cost of transportation equipment construction can be reduced. 2) The amount of computation is dispersed, so it has better real-time and scalability. However, the distributed driving strategy can only obtain a suboptimal solution rather than the global optimal solution [14]. Under this strategy, vehicles will pass through intersections on a first-come-first-served basis, and some adjustments may be made based on the specific control method [54].

Cooperative game theory is a suitable tool for modeling strategic interaction between autonomous driving vehicles and it has been exploited for vehicle interactions at intersections by a lot of researchers. In [1], a multi-vehicle interaction strategy based on the leader-follower game is proposed. According to specific rules, the ego-vehicle determines the leader-follower relationship with other vehicles to make the corresponding operation. In [55], a vehicle interactive reasoning model based on fuzzy logic is established. A conflict decision model of intelligent vehicles based on the game theory is proposed. In [56], an autonomous driving strategy based on the cooperative game theory is proposed to avoid vehicle collisions at intersections. In [57], a cooperative decision-making method based on the coalitional game theory is proposed to solve the multi-lane merging problem for autonomous driving vehicles.

Model predictive control is a special optimal control method and has been widely studied in recent years. It calculates the future multi-step control quantity, but the autonomous vehicle only executes the latest one in each control cycle. The model predictive control will be carried out again in the next cycle. In this way, the autonomous vehicle can deal with emergencies immediately. In [58], a distributed MPC algorithm is used to realize the multi-vehicle cooperative traffic, which improves the traffic efficiency of the intersection. In [13], autonomous driving at intersections is realized according to the first-come-first-served traffic strategy and combined with virtual platooning control. In [59], the intersection control problem without signal is modeled as a distributed optimal control problem.

\section{B. The Individual Driving Strategy}

Although the cooperative driving strategy has considerable advantages, there are also some problems. 1) The cooperative driving strategy relies too much on wireless communication. Once there is a large delay or error in communication, it will have a catastrophic impact on the safety of intersection traffic. 2) Most participants will also be human-driving vehicles in the near future and it is difficult for autonomous driving vehicles to establish robust communication with human drivers. Thus, the research on individual driving strategies is also valuable. The individual driving strategy means that the autonomous vehicle can take appropriate decisions independently without using the V2I or V2V technology, mainly depends on itself.

\section{1) The Classical Strategy}

The representative classical strategy is the state machine model. Finite State Machine (FSM) divides the state of vehicles into a limited number of categories. When the external scenario changes, the autonomous driving vehicle takes corresponding measures according to the predefined state change rules. Hierarchical State Machine (HSM) is an improvement on the basis of FSM. It divides original parallel classes into several layers to improve maintainability and expandability. Thanks to its good stability and easy operation, the state machine model has been widely used. For instance, many teams in the DARPA challenge have adopted the HSM as their decision-making method [60, 61]. In [12], the FSM is considered to use at the T-intersection. However, the classical method is more suitable for simple scenarios rather than complex dynamic scenarios, because the artificially defined rules cannot adapt to all situations [62].

\section{2) The Learning-based Strategy}

The learning-based strategy has many detailed categories including statistic learning, deep learning, reinforcement learning, imitation learning, transfer learning, etc. Compared with the classical strategy, the learning-based strategy uses a large amount of data for training to obtain decision-making ability in a complex dynamic environment. In [63], Hidden Markov Model (HMM) is used to predict the intention of other vehicles at intersections, and the Partially Observable 
Markov Decision Process (POMDP) method is used to make decisions. In [64], an intention inference method of vehicles based on the Support Vector Machine (SVM) has been proposed. In [65], reinforcement learning is proposed to achieve autonomous driving at intersections. In [66], the transfer learning method is used to classify intersections. In [67], an autonomous driving simulation experiment is carried out through conditional imitation learning. In essence, the learning-based strategy is more suitable for complex dynamic scenarios. However, the learning-based strategy has high training costs and is difficult to realize semantic interpretation Recently, some researchers focus on interpretable learning algorithms and lifelong learning algorithms to solve the above shortcomings [68, 69].

\section{PROBLEMS AND FutURE OUTLOOKS}

In this section, we focus on the problems of the current autonomous driving strategies as well as the future research outlooks at intersections.

\section{A. Problems}

Although some useful methods of autonomous driving at intersections have been brought up or tested, there are still some problems to be addressed. Firstly, when the number of vehicles increases, how to ensure the real-time and robustness of the algorithm is a great challenge. Most of the existing autonomous driving strategies at intersections rely on $\mathrm{V} 2 \mathrm{~V}$ or V2I technology. In this case, it is of great significance to ensure the algorithm is still safe under the condition of certain sensing noise and communication delay. In addition, most of the above strategies are based on scenarios that all the participants are autonomous driving vehicles, ignoring highly complex participants such as human-driving vehicles and pedestrians. How to ensure smooth and safe traffic in such a complex situation with many participants is really difficult.

\section{B. Future Outlooks}

For now, the autonomous driving vehicle is not as flexible as the vehicle driven by an experienced human driver when dealing with a complex dynamic environment. For example, autonomous vehicles may be in deadlocks or collisions because of a failure to understand the interactions [70]. To improve the level of autonomous driving at intersections, we have listed some future outlooks worthy of study here.

\section{1) The Cognition-based Driving Strategy}

The main reason why autonomous vehicles cannot perform well in complex dynamic scenes is that they have not formed a good knowledge and understanding of the environment. The incomprehension of the environment contains two important aspects, one is the incomprehension of the input information, and the other is the incomprehension of the decision-making behavior. More advanced autonomous driving strategies should incorporate stronger cognitive abilities. The cognitive ability includes the understanding of the environmental semantics, the understanding of driving behavior, the interpretability of algorithms, and so on.

\section{2) The Transfer-based Driving Strategy}

Sometimes, autonomous driving vehicles may deadlock or collide in a complex dynamic environment. The former is often because the algorithm is too conservative, while the latter is often because the algorithm is too aggressive. However, an experienced human driver can accurately analyze the intentions of other participants and flexibly make appropriate actions in this situation. In recent years, some researchers have paid attention to driver behavior transfer or imitating driver's actions based on the learning-based approach [71-74]. This is a promising research direction and needs further research.

\section{3) The Interaction-based Driving Strategy}

As we know, the reason why the intersection is more challenging for autonomous driving vehicles than other scenarios is that it has greater dynamic characteristics and uncertainty. In addition to other autonomous driving vehicles, there are also participants such as human-driving vehicles and pedestrians near the intersection, and their actions are sometimes irregular and even violate traffic laws. To better capture the intention of surrounding dynamic participants, it is necessary to establish interaction with them. The prediction of dynamic participants around the autonomous vehicle has become the focus of some researchers [75-80]. In addition, vehicle-to-pedestrian (V2P) is also a promising way [81].

\section{4) The Fusion-based Driving Strategy}

Each method has its own shortcomings, and the fusion of methods is an effective method to improve the robustness of the system. For example, the learning-based driving strategy can be combined with the rule-based driving strategy, and the centralized driving strategy can be combined with the distributed driving strategy. Also, multiple methods in the same driving strategy can also be combined. In the perception level of the autonomous driving framework, multi-sensor fusion has been widely used [82]. The integration of multiple driving strategies is conducive to complement each other's deficiencies to improve overall performance. So, why not apply this integration strategy to the decision-making and planning levels as well?

\section{CONCLUSION}

As an important traffic element, the intersection plays a crucial role in the efficiency, safety, and energy consumption of the whole traffic. In this article, we focus on autonomous driving at intersections and make an overview. In summary, the intersection is still a challenging scenario for autonomous driving. With the continuous maturity of technologies such as cognition, prediction, and networking, we believe that traffic at intersections will be greatly improved.

\section{REFERENCES}

[1] N. Li, Y. Yao, I. Kolmanovsky, E. Atkins, and A. R. Girard, "Game-theoretic modeling of multi-vehicle interactions at uncontrolled intersections," IEEE Transactions on Intelligent Transportation Systems, pp. 1-15, 2020.

[2] C. Zhao, L. Li, X. Pei, Z. Li, F.-Y. Wang, and X. Wu, "A comparative study of state-of-the-art driving strategies for autonomous vehicles," Accident Analysis \& Prevention, vol. 150, p. 105937, 2021/02/01/ 2021.

[3] M. Aldabbagh, A. Al-Sherbaz, and S. Turner, "The impact of road intersection topology on traffic congestion in urban cities," 2019, pp. 1196-1207. 
[4] F. Lian, B. Chen, K. Zhang, L. Miao, J. Wu, and S. Luan, "Adaptive traffic signal control algorithms based on probe vehicle data," Journal of Intelligent Transportation Systems, vol. 25, pp. 1-17, 05/18 2020.

[5] S. M. A. Shabestary and B. Abdulhai, "Deep learning vs. discrete reinforcement learning for adaptive traffic signal control," in 2018 21 st International Conference on Intelligent Transportation Systems (ITSC), 2018, pp. 286-293.

[6] M. A. S. Kamal, J. Imura, T. Hayakawa, A. Ohata, and K. Aihara, "A vehicle-intersection coordination scheme for smooth flows of traffic without using traffic lights," IEEE Transactions on Intelligent Transportation Systems, vol. 16, no. 3, pp. 1136-1147, 2015.

[7] M. S. Shirazi and B. T. Morris, "Looking at intersections: A survey of intersection monitoring, behavior and safety analysis of recent studies," IEEE Transactions on Intelligent Transportation Systems, vol. 18, no. 1, pp. 4-24, 2017

[8] G. Velasco-Hernandez, E. Caicedo-Bravo, J. Barry, and J. Walsh, "Intersection management systems and internet of things: A review," in 2020 IEEE 16th International Conference on Intelligent Computer Communication and Processing (ICCP), 2020, pp. 439-445.

[9] E. Namazi, J. Li, and C. Lu, "Intelligent intersection management systems considering autonomous vehicles: A systematic literature review," IEEE Access, vol. 7, pp. 91946-91965, 2019.

[10] L. Hu, J. Ou, J. Huang, Y. Chen, and D. Cao, "A review of research on traffic conflicts based on intelligent vehicles," IEEE Access, vol. 8, pp. 24471-24483, 2020.

[11] M. W. Levin and D. Rey, "Conflict-point formulation of intersection control for autonomous vehicles," Transportation Research Part C: Emerging Technologies, vol. 85, pp. 528-547, 2017.

[12] Y. Chen, J. Zha, and J. Wang, "An autonomous T-intersection driving strategy considering oncoming vehicles based on connected vehicle technology," IEEE/ASME Transactions on Mechatronics, vol. 24, no. 6, pp. 2779-2790, 2019

[13] A. I. M. Medina, N. v. d. Wouw, and H. Nijmeijer, "Cooperative intersection control based on virtual platooning," IEEE Transactions on Intelligent Transportation Systems, vol. 19, no. 6, pp. 1727-1740, 2018

[14] H. Xu, Y. Zhang, L. Li, and W. Li, "Cooperative driving at unsignalized intersections using tree search," IEEE Transactions on Intelligent Transportation Systems, vol. 21, no. 11, pp. 4563-4571, 2020.

[15] H. Pei, Y. Zhang, Q. Tao, S. Feng, and L. Li, "Distributed cooperative driving in multi-intersection road networks," IEEE Transactions on Vehicular Technology, pp. 1-1, 2021

[16] Y. Hou, G. Wang, and Y. Zhou, "Virtual-Grid Based Traffic Control Strategy With Multiple Intersections Collaboration," IEEE Access, vol. 6, pp. 40105-40119, 2018

[17] H. Zhao, A. Cui, S. A. Cullen, B. Paden, M. Laskey, and K. Goldberg, "Fluids: A first-order local urban intersection driving simulator," in CASE: IEEE, 2018

[18] M. Fellendorf and P. Vortisch, "Microscopic traffic flow simulator VISSIM," 2011, pp. 63-93.

[19] P. A. Lopez et al., "Microscopic traffic simulation using SUMO," in 2018 21st International Conference on Intelligent Transportation Systems (ITSC), 2018, pp. 2575-2582.

[20] Z. Wu, J. Yang, and L. Huang, "Study on the collision avoidance strategy at unsignalized intersection based on PreScan simulation," Procedia - Social and Behavioral Sciences, vol. 96, pp. 1315-1321, 2013/11/06/ 2013.

[21] A. Dosovitskiy, G. Ros, F. Codevilla, A. Lopez, and V. Koltun, "CARLA: An open urban driving simulator," in Conference on robot learning, 2017, pp. 1-16: PMLR.

[22] Y. Sun and X. Fan, "Research on the application of CarSim in vehicle simulation and development," International Journal, vol. 4, 2017.

[23] P. Cai, Y. Lee, Y. Luo, and D. Hsu, "SUMMIT: A simulator for urban driving in massive mixed traffic," in 2020 IEEE International Conference on Robotics and Automation (ICRA), 2020, pp. 4023-4029.

[24] G. Rong et al., "Lgsvl simulator: A high fidelity simulator for autonomous driving," in 2020 IEEE 23rd International Conference on Intelligent Transportation Systems (ITSC), 2020, pp. 1-6: IEEE.

[25] J. Xu et al., "An automated learning-based procedure for large-scale vehicle dynamics modeling on baidu apollo platform," in 2019
IEEE/RSJ International Conference on Intelligent Robots and Systems (IROS), 2019, pp. 5049-5056: IEEE.

[26] S. Shah, D. Dey, C. Lovett, and A. Kapoor, "Airsim: High-fidelity visual and physical simulation for autonomous vehicles," in Field and service robotics, 2018, pp. 621-635: Springer.

[27] B. Schwab, C. Beil, and T. H. Kolbe, "Spatio-semantic road space modeling for vehicle-pedestrian simulation to test automated driving systems," Sustainability, vol. 12, no. 9, p. 3799, 2020.

[28] F. Zhou, Y. Liu, and R. Chen, "Research on collaborative innovation of intelligent connected vehicles industry based on test field: Embedded case study from the perspective of open innovation," Sustainability, vol. 13, no. 11, p. 5880, 2021

[29] D. S. Buse, M. Schettler, N. Kothe, P. Reinold, C. Sommer, and F Dressler, "Bridging worlds: Integrating hardware-in-the-loop testing with large-scale VANET simulation," in 2018 14th Annual Conference on Wireless On-demand Network Systems and Services (WONS), 2018, pp. 33-36.

[30] H. Kaths, A. Keler, J. Kaths, and F. Busch, "Analyzing the behavior of bicyclists using a bicycle simulator with a coupled SUMO and DYNA4 simulated environment," in SUMO, 2019, pp. 199-205.

[31] E. Dovgan, "Multiobjective discovery of driving strategies using the SCANeR Studio," Dep. Intell. Syst, 2017.

[32] L. Zhang, Z. Du, S. Zhao, Y. Zhai, and Y. Shen, "Development and verification of traffic confrontation simulation test platform based on PanoSim," in 2020 IEEE 4th Information Technology, Networking, Electronic and Automation Control Conference (ITNEC), 2020, vol. 1, pp. 1814-1818.

[33] F. Wang, C. Ye, Y. Zhang, Y. Li, and S. Journal, "Simulation analysis and improvement of the vehicle queuing system on intersections based on MATLAB," The Open Cybernetics, vol. 8, no. 1, 2014.

[34] J. Guo, U. Kurup, and M. Shah, "Is it safe to drive? An overview of factors, metrics, and datasets for driveability assessment in autonomous driving," IEEE Transactions on Intelligent Transportation Systems, vol. 21, no. 8, pp. 3135-3151, 2020.

[35] A. Zyner, S. Worrall, and E. M. Nebot, "ACFR five roundabouts dataset: Naturalistic driving at unsignalized intersections," IEEE Intelligent Transportation Systems Magazine, vol. 11, no. 4, pp. 8-18, 2019.

[36] M. B. Jensen, A. Møgelmose, and T. B. Moeslund, "Presenting the Multi-view Traffic Intersection Dataset (MTID): A Detailed Traffic-Surveillance Dataset," in 2020 IEEE 23rd International Conference on Intelligent Transportation Systems (ITSC), 2020, pp. $1-6$.

[37] Z. Zhu, D. Liang, S. Zhang, X. Huang, B. Li, and S. Hu, "Traffic-sign detection and classification in the wild," in Proceedings of the IEEE conference on computer vision and pattern recognition, 2016, pp. 2110-2118

[38] C. Ertler, J. Mislej, T. Ollmann, L. Porzi, G. Neuhold, and Y. Kuang, "The mapillary traffic sign dataset for detection and classification on a global scale," in European Conference on Computer Vision, 2020, pp. 68-84: Springer.

[39] X. Huang et al., "The ApolloScape dataset for autonomous driving," in 2018 IEEE/CVF Conference on Computer Vision and Pattern Recognition Workshops (CVPRW), 2018, pp. 1067-10676.

[40] G. Neuhold, T. Ollmann, S. Rota Bulo, and P. Kontschieder, "The mapillary vistas dataset for semantic understanding of street scenes," in Proceedings of the IEEE International Conference on Computer Vision, 2017, pp. 4990-4999.

[41] A. Geiger, P. Lenz, C. Stiller, and R. Urtasun, "Vision meets robotics: The KITTI dataset," The International Journal of Robotics Research, vol. 32, no. 11, pp. 1231-1237, 2013/09/01 2013.

[42] M. Cordts et al., "The Cityscapes Dataset for Semantic Urban Scene Understanding," 2016 IEEE Conference on Computer Vision and Pattern Recognition (CVPR), pp. 3213-3223, 2016.

[43] A. Rasouli, I. Kotseruba, and J. K. Tsotsos, "Are they going to cross? A benchmark dataset and baseline for pedestrian crosswalk behavior," in 2017 IEEE International Conference on Computer Vision Workshops (ICCVW), 2017, pp. 206-213.

[44] F. Yu et al., "Bdd100k: A diverse driving dataset for heterogeneous multitask learning," in Proceedings of the IEEE/CVF conference on computer vision and pattern recognition, 2020, pp. 2636-2645. 
[45] A. Patil, S. Malla, H. Gang, and Y.-T. Chen, "The h3d dataset for full-surround $3 \mathrm{~d}$ multi-object detection and tracking in crowded urban scenes," in 2019 International Conference on Robotics and Automation (ICRA), 2019, pp. 9552-9557: IEEE.

[46] H. Caesar et al., "Nuscenes: A multimodal dataset for autonomous driving," in Proceedings of the IEEE/CVF conference on computer vision and pattern recognition, 2020, pp. 11621-11631.

[47] J. Gregoire, "Priority-based coordination of mobile robots," 10/03 2014.

[48] T. Zhou, H. Zhao, Y. Wang, and J. Yang, "A multi-vehicles optimization method for intersection cooperative driving," in 2017 29th Chinese Control And Decision Conference (CCDC), 2017, pp. 5855-5861.

[49] J. Wu, F. Perronnet, and A. Abbas-Turki, "Cooperative vehicle-actuator system: A sequencebased framework of cooperative intersections management," IET Intelligent Transport Systems, vol. 8, $08 / 212013$

[50] L. Qian, C. Chen, B. Wu, L. Xuan, and T. Wang, "Optimal control of connected and automated vehicles at unsignalized intersections: discrete and regroup," in 2020 International Conference on Intelligent Engineering and Management (ICIEM), 2020, pp. 370-375.

[51] H. Hu, S. F. Smith, and R. Goldstein, "Cooperative schedule-driven intersection control with connected and autonomous vehicles," in 2019 IEEE/RSJ International Conference on Intelligent Robots and Systems (IROS), 2019, pp. 1668-1673.

[52] B. Li, Y. Zhang, Y. Zhang, N. Jia, and Y. Ge, "Near-optimal online motion planning of connected and automated vehicles at a signal-free and lane-free intersection," in 2018 IEEE Intelligent Vehicles Symposium (IV), 2018, pp. 1432-1437.

[53] W. Zhao, D. Ngoduy, S. Shepherd, R. Liu, and M. Papageorgiou, "A platoon based cooperative eco-driving model for mixed automated and human-driven vehicles at a signalised intersection," Transportation Research Part C: Emerging Technologies, vol. 95, pp. 802-821, 2018.

[54] Y. Meng, L. Li, F. Wang, K. Li, and Z. Li, "Analysis of cooperative driving strategies for nonsignalized intersections," IEEE Transactions on Vehicular Technology, vol. 67, no. 4, pp. 2900-2911, 2018.

[55] X. Chen, Y. Sun, Y. Ou, X. Zheng, Z. Wang, and M. Li, "A conflict decision model based on game theory for intelligent vehicles at urban unsignalized intersections," IEEE Access, vol. 8, pp. 189546-189555, 2020.

[56] Z. Yang, H. Huang, G. Wang, X. Pei, and Y. Danya, "Cooperative driving model for non-signalized intersections with cooperative games," Journal of Central South University, vol. 25, no. 9, pp. 2164-2181, 2018/09/01 2018.

[57] P. Hang, C. Lv, C. Huang, Y. Xing, and Z. Hu, "Cooperative decision making of connected automated vehicles at multi-lane merging zone: A coalitional game approach," IEEE Transactions on Intelligent Transportation Systems, 2021.

[58] A. Katriniok, P. Kleibaum, and M. Joševski, "Distributed model predictive control for intersection automation using a parallelized optimization approach," IFAC-PapersOnLine, vol. 50, no. 1, pp. 5940-5946, 2017/07/01/ 2017.

[59] Y. Zhang, A. A. Malikopoulos, and C. G. Cassandras, "Decentralized optimal control for connected automated vehicles at intersections including left and right turns," in 2017 IEEE 56th Annual Conference on Decision and Control (CDC), 2017, pp. 4428-4433.

[60] L. Fu, A. Yazici, and U. Ozguner, "Route planning for OSU-ACT autonomous vehicle in DARPA urban challenge," in 2008 IEEE Intelligent Vehicles Symposium, 2008, pp. 781-786.

[61] M. Montemerlo et al., "Junior: The stanford entry in the urban challenge," Journal of Field Robotics, vol. 25, pp. 569-597, 09/01 2008.

[62] X. Lin, J. Zhang, J. Shang, Y. Wang, H. Yu, and X. Zhang, "Decision making through occluded intersections for autonomous driving," in 2019 IEEE Intelligent Transportation Systems Conference (ITSC), 2019 , pp. 2449-2455.

[63] W. Song, G. Xiong, and H. Chen, "Intention-aware autonomous driving decision-making in an uncontrolled intersection,"
Mathematical Problems in Engineering, vol. 2016, p. 1025349, 2016/04/28 2016.

[64] Y. Jeong, K. Yi, and S. Park, "SVM based Intention Inference and Motion Planning at Uncontrolled Intersection," IFAC-PapersOnLine, vol. 52, no. 8, pp. 356-361, 2019/01/01/ 2019.

[65] X. Ma, J. Li, M. J. Kochenderfer, D. Isele, and K. Fujimura, "Reinforcement learning for autonomous driving with latent state inference and spatial-temporal relationships," ICRA, 2021.

[66] U. Baumann, Y.-Y. Huang, C. Gläser, M. Herman, H. Banzhaf, and J. M. Zöllner, "Classifying road intersections using transfer-learning on a deep neural network," in 2018 21st International Conference on Intelligent Transportation Systems (ITSC), 2018, pp. 683-690: IEEE.

[67] F. Codevilla, M. Müller, A. López, V. Koltun, and A. Dosovitskiy, "End-to-end driving via conditional imitation learning," in 2018 IEEE International Conference on Robotics and Automation (ICRA), 2018 , pp. 4693-4700.

[68] J. Chen, S. E. Li, and M. Tomizuka, "Interpretable end-to-end urban autonomous driving with latent deep reinforcement learning," IEEE Transactions on Intelligent Transportation Systems, 2021.

[69] B. Liu, "Learning on the job: Online lifelong and continual learning," in Proceedings of the AAAI Conference on Artificial Intelligence, 2020, vol. 34, no. 09, pp. 13544-13549.

[70] N. Li, I. Kolmanovsky, A. Girard, and Y. Yildiz, Game theoretic modeling of vehicle interactions at unsignalized intersections and application to autonomous vehicle control. 2018.

[71] Z. Li, B. Wang, J. Gong, T. Gao, C. Lu, and G. Wang, "Development and evaluation of two learning-based personalized driver models for pure pursuit path-tracking behaviors," in 2018 IEEE Intelligent Vehicles Symposium (IV), 2018, pp. 79-84.

[72] Z. Li et al., "Transferable driver behavior learning via distribution adaption in the lane change scenario*," in 2019 IEEE Intelligent Vehicles Symposium (IV), 2019, pp. 193-200.

[73] Z. Li, J. Gong, C. Lu, and J. Xi, "Importance weighted gaussian process regression for transferable driver behaviour learning in the lane change scenario," IEEE Transactions on Vehicular Technology, vol. 69 , no. 11 , pp. $12497-12509,2020$.

[74] C. Gong, Z. Li, C. Lu, J. Gong, and F. Hu, "A comparative study on transferable driver behavior learning methods in the lane-changing scenario," in 2019 IEEE Intelligent Transportation Systems Conference (ITSC), 2019, pp. 3999-4005.

[75] J. Li, H. Ma, and M. Tomizuka, "Conditional generative neural system for probabilistic trajectory prediction. In in 2019 IEEE," in RSJ International Conference on Intelligent Robots and Systems (IROS). IEEE, 2019, vol. 2.

[76] K. Chen, X. Song, and X. Ren, "Modeling social interaction and intention for pedestrian trajectory prediction," Physica A: Statistical Mechanics and its Applications, vol. 570, p. 125790, 2021.

[77] Z. Li, J. Gong, C. Lu, and Y. Yi, "Interactive behaviour prediction for heterogeneous traffic participants in the urban road: A graph neural network-based multi-task learning framework," IEEE/ASME Transactions on Mechatronics, 2021.

[78] R. Quan, L. Zhu, Y. Wu, and Y. Yang, "Holistic LSTM for pedestrian trajectory prediction," IEEE transactions on image processing, vol. 30, pp. 3229-3239, 2021.

[79] C. L. Zirui Li, Yangtian Yi, Jianwei Gong, "A hierarchical framework for interactive behaviour prediction of heterogeneous traffic participants based on graph neural network," IEEE Transactions on Intelligent Transportation Systems, 2021.

[80] J. Li, F. Yang, M. Tomizuka, and C. Choi, "Evolvegraph: Multi-agent trajectory prediction with dynamic relational reasoning," Proceedings of the Neural Information Processing Systems, 2020.

[81] R. Q. Malik, K. N. Ramli, Z. H. Kareem, M. I. Habelalmatee, A. H. Abbas, and A. Alamoody, "An overview on V2P communication system: Architecture and application," in 2020 3rd International Conference on Engineering Technology and its Applications (IICETA), 2020, pp. 174-178: IEEE.

[82] S. A. Kashinath et al., "Review of data fusion methods for real-time and multi-sensor traffic flow analysis," IEEE Access, 2021. 